\title{
Estimación de tasas de compresión de imágenes mediante métricas de distorsión completamente referenciadas
}

\author{
Julio-César García*\$, Germán Castellanos* \\ * Departamento de Ingeniería Eléctrica, Electrónica y de Computación, \\ Universidad Nacional de Colombia Sede Manizales, Manizales, Colombia \\ §e-mail:jcgarciaa@unal.edu.co
}

(Recibido: Noviembre 14 de 2007 - Aceptado: Junio 11 de 2008)

\begin{abstract}
Resumen
El acceso remoto a las imágenes es un área de creciente interés debido a la necesidad de almacenar, procesar y visualizar una gran cantidad de imágenes disponibles en portales y servidores alrededor del mundo. Esta necesidad se vuelve un desafío a medida que aumentan el tamaño y la calidad de las imágenes. El presente trabajo presenta un método para la estimación de tasas de compresión de imágenes mediante métricas de distorsión completamente referenciadas e ilustra su aplicación a imágenes médicas que son procesadas por las técnicas JPEG, SPIHT y EBCOT. Este método hace posible que imágenes de alta definición puedan ser transmitidas en tiempos más cortos. Las tasas de compresión de las tres técnicas mencionadas se calculan considerando los valores límite de compresión sin pérdida de la percepción y los valores de correlación entre medidas. Los resultados obtenidos en este trabajo demuestran que es posible lograr la transmisión progresiva de imágenes para cada una de las tres técnicas, manteniendo la misma calidad de percepción y acortando los tiempos de transmisión.
\end{abstract}

Palabras clave: Métricas de distorsión, Métricas completamente referenciadas, Niveles de calidad, JPEG, JPEG2000, SPIHT.

ELECTRONICS ENGINEERING

\section{Estimation of image compression rates by means of full-referenced distortion metrics}

\begin{abstract}
Image remote access is an area of increasing interest because of the need to store, process and display a large amount of images available in web portals and network servers around the world. This need becomes a challenge as the size and quality of images increase. This work presents a method for the estimation of compression rates of images by means of full-referenced distortion metrics and shows its application to medical images that are processed by the JPEG, SPIHT, and EBCOT techniques. This method makes it possible that high-definition images can be transmitted in shorter times. Compression rates for the three mentioned techniques are calculated by considering the perceivedlossless compression limits and the correlation values between measurements. The results obtained in this work show that it is possible to achieve the progressive transmission of images for each of the three techniques, keeping the same perceived quality and shortening the transmission times.
\end{abstract}

Keywords: Distortion metrics, Full-referenced metrics, Quality levels, JPEG, JPEG2000, SPIHT. 


\section{Introducción}

La transmisión de imágenes médicas se ha enfocado a la visualización de imágenes de alta definición a través de anchos de banda reducidos. Las imágenes de alta definición se envían de tal manera que permitan que el experto pueda revisarlas sin presentar espera alguna en el momento de la transmisión y, que en el momento de visualizar las imágenes, el experto tenga la confianza de tener imágenes de calidad suficiente para emitir un diagnóstico. Aplicaciones como mapas satelitales, microscopía de alta resolución y cristalografía, requieren de imágenes de alta calidad y definición, tal como lo han mostrado Dudek et al. (2007) y Kosheleva (2004). La compresión de imágenes mediante esquemas como JPEG2000 (desarrollado por Taubman, 2000), ha permitido el almacenamiento masivo y su rápido acceso, tanto para imágenes de alta resolución y tamaño, como para bases de datos de grandes cantidades de imágenes. En efecto, un esquema de acceso a imágenes involucra la compresión de las mismas con estimación de tasas. Si la imagen $X_{i}$ es transmitida de manera completa, se espera que la tasa de transmisión sea equivalente a la cantidad de información original de la imagen por unidad de tiempo. Sin embargo, para imágenes de alta definición, la tasa de transmisión hace que el tiempo de transmisión sea intolerablemente largo para el servicio. Para esto, se necesita que la imagen sea comprimida a una cantidad $L_{i}$ menor que la cantidad de información original. En efecto, mediante técnicas de codificación entrópica, es posible obtener tasas de transmisión menores, soportables para ciertos valores exigidos.

El objetivo es determinar un método de transmisión de imágenes, de tal manera que en el momento de realizar la codificación, puedan enviarse de manera inmediata los valores relevantes de la imagen, que son de utilidad al experto. Esto implica un esquema de transmisión progresiva, lo cual aplica a las tres técnicas de compresión evaluadas en este trabajo: Fourier (aplicado a la técnica JPEG), partición espacial mediante árboles jerárquicos (SPIHT) y bloques de código embebidos mediante optimización truncada (EBCOT). Aunque en la literatura se han hecho por separado los análisis de calidad para cada técnica, muchos centros de teleconsulta han optado por realizar la transmisión utilizando la técnica JPEG, debido a su uso masivo y a la facilidad de adquisición en dispositivos como cámaras digitales, como lo ilustran $\mathrm{Zhu} \& \mathrm{Wu}$ (2005).

El objetivo de este trabajo es demostrar que la transmisión utilizando un esquema basado en capas de calidad, como la codificación de bloques embebidos con optimización truncada (EBCOT), produce mejores resultados que el esquema JPEG, basado en índices de calidad. Aunque se han desarrollado técnicas para el mejoramiento de la calidad de las imágenes transmitidas usando JPEG, tal como lo ilustran Chen \& Hao (2004), estas técnicas producen un procesamiento adicional y la inclusión de artefactos artificiales indeseables para los expertos. La codificación basada en onduletas (wavelets) provee mejoras en la calidad de la imagen a tasas de compresión altas, y es más robusta respecto a errores de transmisión y decodificación, además de facilitar la transmisión progresiva de imágenes.

Lewis \& Knowles (1992) y Kovacevic \& Sweldens (2000) han desarrollado e implementado una variedad de esquemas para compresión de imágenes basadas en onduletas. En muchas aplicaciones, los esquemas basados en onduletas (sub-band coding) presentan ventajas sobre otros esquemas de codificación, como la transformada discreta del coseno $(D C T)$. La norma JPEG2000 no solamente suministra mejores tasas de compresión, sino que además ofrece nuevas características en comparación con las técnicas de compresión de imágenes existentes. Algunas de las características soportadas por JPEG2000 incluyen: compresión con y sin pérdidas, compresión en tonos continuos y de dos niveles, transmisión progresiva con ajuste de resolución y precisión de elementos, codificación de regiones de interés, procesamiento de las regiones comprimidas y robustez al error, tal como lo han implementado Gangadhar \& Bhatia (2005).

En la Sección 2, se explica la metodología, los modelos de estimación de la tasa de compresión (de acuerdo con el contenido del mapa de transformación de la imagen), las métricas 
utilizadas para la estimación, y se presenta un ejemplo ilustrativo para imágenes radiológicas. En la Sección 3, se presentan resultados experimentales para el método propuesto y en la Sección 4 se presentan las conclusiones alcanzadas.

\section{Metodología}

Sea $X_{i}$ una de las imágenes que va a ser transmitida con un valor de calidad $Q_{i}$. Para que $X_{i}$ sea transmitida, se requiere que la cantidad de información contenida sea minimizada. Debido a la característica de alta correlación entre muestras vecinas contenidas, el espectro de $X_{i}$ presenta valores altos en los coeficientes de baja frecuencia, lo que hace posible que, al codificar el mapa de transformación, la cantidad de información pueda ser reducida. Esto es posible mediante algoritmos de codificación aritmética, donde los datos redundantes son aglomerados en conjuntos pequeños de información. Para las técnicas de compresión a evaluar, los datos relevantes, denominados detalles, son minimizados o excluidos, de acuerdo al valor de $Q_{i}$. Entonces, se obtiene que $L_{i}<H\left(X_{i}\right)$, siendo $H\left(X_{i}\right)$ la cantidad media de información de la imagen. A continuación se ilustran las técnicas a evaluar.

La técnica basada en la transformada de Fourier-DCT utiliza la transformada discreta del Coseno (DCT):

$$
W[k, l]=\alpha[k] \beta[l] \sum_{m=0}^{M-1} \sum_{n=0}^{N-1} x[m, n] \psi(m, k, N) \psi(n, l, N)
$$

donde

$$
\begin{aligned}
& \psi(m, k, N)=\cos \left[\frac{\pi(2 m+1) k}{2 N}\right] \\
& \psi(n, l, N)=\cos \left[\frac{\pi(2 n+1) l}{2 N}\right]
\end{aligned}
$$

$\operatorname{con} x[m, n] \in X_{i}$. Los coeficientes $W[k, l]$ presentan valores altos para valores de $k, l$ pequeños, siendo el más importante $W[0,0]$, el cual se conoce como componente DC (Salomon, 2004). Lo anterior implica que para reducir $L_{i}$ es posible truncar los coeficientes correspondientes a valores grandes de $k$, $l$, de acuerdo al valor de $Q_{i}$.
Para efectos de percepción, $X_{i}$ es dividida en bloques de código $C_{i} \subset X_{i}$ no traslapados y a cada una se le aplica la transformación y posterior codificación.

La técnica de partición espacial mediante árboles jerárquicos (SPIHT) utiliza la transformada de onduleta(DWT):

$$
W[k, l, d l]=\sum_{m=0}^{M-1} \sum_{n=0}^{N-1} x[m, n] \psi(m, k, N, d l) \psi(n, l, N, d l)
$$

donde $\psi(m, k, N)$ y $\psi(n, l, N)$ corresponden a un filtro de onduleta, el cual descompone cada elemento $x[m, n]$ en valores de resolución espacial $d l$ denominados niveles de descomposición, lo que permite que para $x[m, n]$ existan varios representantes de energía en diferentes niveles. Esto implica que para valores $d l$ bajos, se presentan sus componentes en alta frecuencia (los cuales se conocen como coeficientes de detalle) y para valores $d l$ altos, se presentan sus componentes en baja frecuencia (los cuales se conocen como coeficientes de aproximación). De igual manera que en la técnica anterior, y debido a la propiedad de alta correlación entre elementos vecinos de la imagen, los valores $W[k, l, d l]$ con $k, l, d l$ bajos, son los más importantes, por lo que la reducción de $L_{i}$ se realiza truncando los coeficientes $W[k, l, d l]$ menos importantes, permitiendo una reducción de tamaño en la codificación. La partición espacial se hace ubicando las posiciones $[m, n]$ que obtuvieron los coeficientes $W[k, l, d l]$ más importantes.

La técnica de codificación de bloques embebidos mediante optimización truncada (EBCOT) se diferencia de la técnica SPIHT en que no se utilizan los coeficientes más importantes de la imagen completa para la codificación. El mapa de onduleta de la imagen $W_{i}$ es dividido en bloques de código $C_{b} \subset W_{i}$ de tal manera que cada coeficiente $c[k, l] \in C_{b}$ es codificado, por lo que cada región tiene su propia cantidad $L_{b}$. Lo anterior permite que se presenten diferentes niveles de detalle $Q_{i}$ (denominados niveles de calidad), de tal manera que se trunquen los bloques correspondientes a los coeficientes menos importantes. La tasa de transmisión $R_{i}$ determina el tiempo de transmisión 


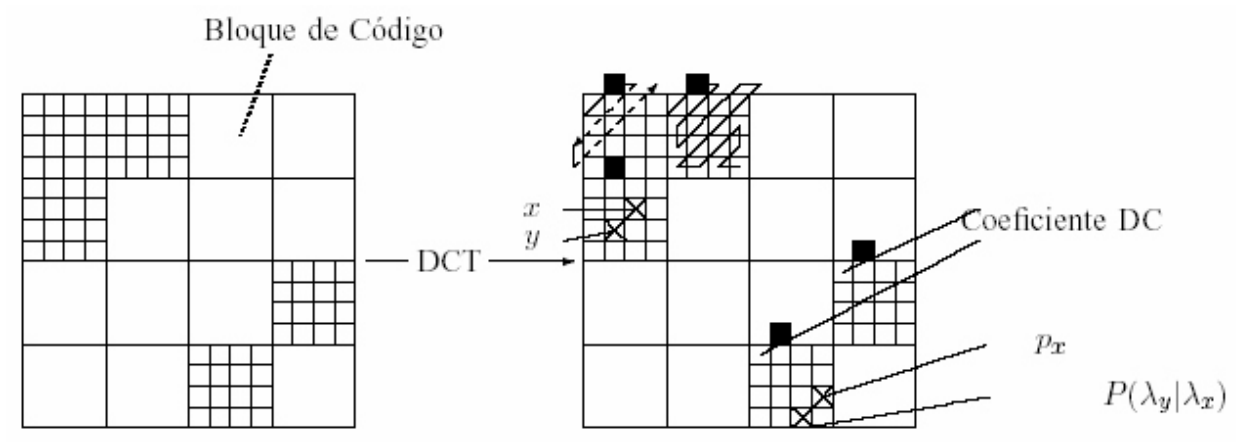

Figura 1. Estimación de segundo orden de la tasa de compresión en imágenes.

de la $X_{i}$; en efecto, para el caso ideal, se tiene que $t=L_{i} / R_{i}$. Lo anterior significa que, si $L_{i}<R_{i}$, el tiempo de transmisión se reduce. La estimación de la tasa de compresión es importante para determinar si es factible enviar una imagen o porción de la imagen en un tiempo exigido $t=T$. Diferentes modelos son utilizados para estimar el valor de $L_{i}$ en bits por pixel, de acuerdo con las características de la imagen. El procedimiento de cálculo se ilustra en la Figura 1, donde la imagen se divide en códigos de bloque, para posteriormente calcular las probabilidades de ocurrencia. Los modelos serán descritos a modo de ilustración, con el fin de dar una idea a priori del modelo que se va a utilizar.

En el modelo de orden cero (ZOM), cada $x[m, n] \in X_{i}$ es estadísticamente independiente y cada objeto o región de interés $F_{\lambda} \subset X_{i}$ tiene una probabilidad igual de ocurrencia. Siendo $\Lambda=\sup (\lambda)$ el número de objetos, la tasa de compresión óptima está dada por:

$$
L_{i}=\log _{2} \Lambda
$$

En el modelo de primer orden (FOM), cada elemento $x[m, n] \in X_{i}$ es estadísticamente independiente y cada objeto o región de interés $F_{\lambda} \subset X_{i}$ tiene una probabilidad $p_{\lambda}$ de ocurrencia. La tasa de compresión óptima está dada por:

$$
L_{i}=\sum_{\lambda=1}^{\Lambda} p_{\lambda} \log _{2} p_{\lambda}
$$

En el modelo de segundo orden (SOM), cada elemento $y[m, n] \in X_{i}$ tiene probabilidad $P\left(\lambda_{y} \mid \lambda_{x}\right)$ de que ocurra, dado que su elemento vecino es $x[m, n] \in X_{i} \quad \mathrm{y}$ cada objeto o región de interés $F_{\lambda} \subset X_{i}$ tiene una probabilidad $p_{\lambda_{x}}$ de ocurrencia. La tasa de compresión óptima está dada por:

$$
L_{i}=\sum_{\lambda_{x}=1}^{\Lambda} p_{\lambda_{x}} \sum_{\lambda_{y}=1}^{\Lambda} P\left(\lambda_{y} \mid \lambda_{x}\right) \log _{2} P\left(\lambda_{y} \mid \lambda_{x}\right)
$$

En el modelo de tercer orden (TOM), cada elemento $z[m, n] \in X_{i}$ tiene una probabilidad de ocurrencia $P\left(\lambda_{z} \mid \lambda_{y}, \lambda_{x}\right)$, dado que sus elementos vecinos son, en su orden, $y, x \in X_{i}$. La tasa de compresión óptima está dada por:

$$
L_{i}=\sum_{\lambda_{x}=1}^{\Lambda} p_{\lambda_{x}} \sum_{\lambda_{y}=1}^{\Lambda} P\left(\lambda_{y} \mid \lambda_{x}\right) \sum_{\lambda_{z}=i}^{\Lambda} P\left(\lambda_{z} \mid \lambda_{y}, \lambda_{x}\right) \log _{2} P\left(\lambda_{z} \mid \lambda_{y}, \lambda_{x}\right)
$$

En el modelo general de Shannon (SGM), si se asume que $B_{\lambda}$ representa los $\lambda$ elementos más importantes de $x[m, n] \in X_{i}$, entonces se tiene que:

$$
L_{i}=\mathrm{H}\left(X_{i}\right)=\lim _{n \rightarrow \infty} \frac{1}{\lambda} \sum P\left(B_{\lambda}\right) \log _{2} P\left(B_{\lambda}\right)
$$

El caso general fue descrito por Shannon \& Weaver (1949).

Las medidas de calidad se refieren a medidas de distorsión que dan cierto grado de información 
sobre cómo se visualiza la imagen. Las medidas completamente referenciadas utilizan tanto la imagen a transmitir $X_{i}$ como la recuperada después del proceso de inversión (transformada inversa $\left.\tilde{X}_{i}\right)$. En efecto, una medida utiliza funciones de la distancia $M_{p}=d\left(X_{i}, \tilde{X}_{i}\right)$. Varios trabajos han evaluado la calidad de las imágenes comprimidas en JPEG y JPEG2000, de forma separada, como lo hicieron Ginesu et al. (2006).

Sin embargo, el análisis de imágenes médicas ha sido orientado al uso de JPEG como norma de compresión, e inclusive muchos servicios de teleconsulta utilizan imágenes con este formato como lo ilustran Krupinski et al. (2003) y Slone et al. (2003). En los trabajos de Azpiroz-Leehan \& Lerallut (2006) y Christopoulos et al. (2000), se ha demostrado que para ciertas imágenes de prueba, JPEG2000 es el mejor esquema para compresión y, en los trabajos de Aminlou \& Fatemi (2005) y Gangadhar \& Bhatia (2005), se han realizado mejoras mediante aceleradores de proceso.

Entonces, el análisis de la calidad de imágenes médicas, comprimidas por JPEG2000, requeriría una nueva evaluación de expertos, y nuevas imágenes, debido a que la compresión JPEG no es reversible, como en el ejemplo ilustrado en Ringl et al. (2006). Este método establece valores de la tasa de compresión para ciertos niveles de calidad en las tres técnicas utilizadas bajo la norma DICOM: JPEG, SPIHT y JPEG2000, utilizando imágenes comprimidas en JPEG, para luego ser procesadas en JPEG2000, con la misma calidad de imagen.

\subsection{Medidas espaciales}

Sean $C_{k}(i, j)$ y $\tilde{C}_{k}(i, j)$ los respectivos coeficientes representativos en el espacio de $X$ y $\tilde{X}$, respectivamente. De acuerdo con Avcibas et al. (2003), las siguientes medidas están en $\mathrm{L}_{2}$ :

Error cuadrático medio,

$$
M_{1}=\frac{1}{K} \sum_{k=1}^{K}\left[\frac{1}{N^{2}} \sum_{i, j=1}^{N}\left|C_{k}(i, j)-\widetilde{C}_{k}(i, j)\right|^{2}\right]^{1 / 2}
$$

Distancia de Czekanowski,

$$
M_{2}=\frac{1}{N^{2}} \sum_{i, j=0}^{N-1}\left[1-\frac{2 \sum_{k=1}^{K} \min \left\{C_{k}(i, j), \widetilde{C}_{k}(i, j)\right\}}{\sum_{k=1}^{K}\left(C_{k}(i, j)+\widetilde{C}_{k}(i, j)\right)}\right]
$$

Fidelidad de la imagen,

$$
M_{3}=1-\left[\frac{1}{K} \sum_{k=1}^{K} \frac{\sum_{i, j=0}^{N-1}\left(C_{k}(i, j)-\widetilde{C}_{k}(i, j)\right)^{2}}{\sum_{i, j=0}^{N-1}\left(C_{k}(i, j)\right)^{2}}\right]
$$

\subsection{Medidas espectrales}

Sean $W_{k}(u, v)$ y $\tilde{W}_{k}(u, v)$ los respectivos coeficientes representativos en el espectro de $X$ y $\widetilde{X}$, respectivamente. A continuación, se presenta una medida espectral:

$$
M_{4}=\frac{1}{K N^{2}} \sum_{k=1}^{K} \sum_{u, v=0}^{N-1}|| W_{k}(u, v)|-| \widetilde{W}_{k}(u, v)||^{2}
$$

La expresión $W_{k}(u, v)$ implica que debe considerarse algún espacio de transformación, debido a que, por ejemplo, la base onduleta no es la misma base de Fourier. De acuerdo con esto, no puede establecerse una comparación entre las técnicas utilizando esta medida, ya que se tendrá un valor más favorable hacia la técnica que utilice la misma base generadora de la medida. Sin embargo, para efectos de estimación de la tasa de compresión para cada técnica, se considerará la variabilidad de la medida, en lugar de la comparación entre valores.

En el momento de la recepción, es necesario comparar la calidad de la imagen visualizada contra la que reside en el servidor, para establecer una realimentación para el control de la tasa de transmisión. Es necesario determinar, como primera medida, los valores límite de calidad, sin utilizar métricas globales de calidad, como lo hacen Dosselmann \& Yang (2005), para valores de 


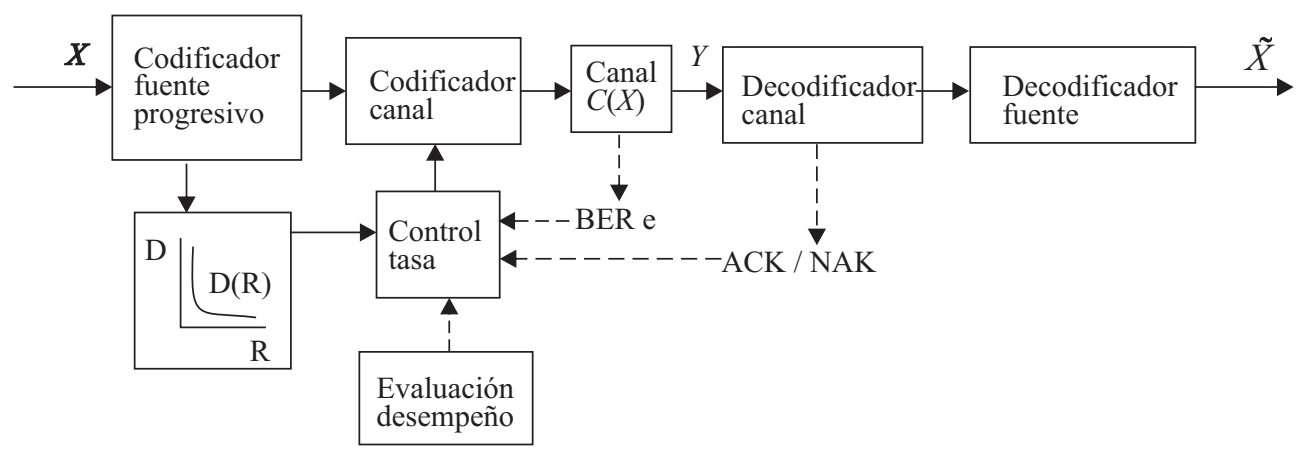

Figura 2. Esquema de transmisión de la imagen X utilizando estimación de la tasa de compresión.

la tasa de compresión determinados en los tres esquemas a evaluar, para un conjunto dado de imágenes. La medida espectral será generada por la base onduleta, por lo que se espera que las técnicas SPIHT y EBCOT presenten valores menores que JPEG, para los valores de tasa estimada dados.

Las técnicas de compresión soportadas por la norma DICOM fueron establecidas para el adecuado almacenamiento, transmisión y caracterización de imágenes médicas provenientes de diferentes fuentes. La Figura 2 ilustra un método de transmisión de imágenes, utilizando información del canal $C(X)$ y el decodificador del canal. En este método, el núcleo principal consiste en un algoritmo que entrega un valor de tasa para una técnica de compresión arbitraria.

La tasa de compresión se estima de la siguiente manera: para $X_{i}$, se tiene un valor predeterminado de calidad perceptual $Q_{i}(\mathrm{O})$. Este valor se usa para alguna técnica de compresión con pérdidas, como JPEG, con base en análisis de concordancia realizados por radiólogos expertos. Este valor hace que la imagen quede comprimida a $L_{i}(\mathrm{O})$. Posteriormente, se aplican las medidas espaciales $M_{1}, M_{2}, M_{3}$ y la espectral $M_{4}$ para las imágenes fuente y estimada con la técnica JPEG. Las medidas se promedian con respecto al conjunto de imágenes comprimidas, así:

$$
M_{p}=\frac{1}{I} \sum_{i=1}^{I} M(i)_{p}=\frac{1}{I} \sum_{i=1}^{I} d\left(X_{i}, \widetilde{X}_{i}\right)
$$

El modelo a utilizar es el de segundo orden, debido a la característica de alta correlación que presenta la imagen entre elementos vecinos. LópezFernández (2002) ilustra una aplicación. Mediante este modelo, se determina el valor de tasa de compresión ideal $L_{i}$ para el conjunto de imágenes $\left\{X_{i}\right\}$ comprimidas bajo el mismo valor perceptual de calidad $Q_{i}(0)$. El valor $L_{i}$ es la tasa de compresión objetivo para las técnicas SPIHT y EBCOT. La imagen $X_{i}$ es comprimida con estas técnicas. Se obtiene el valor de la tasa de compresión $L_{i}$, de modo que exista una cercanía para cada medida de calidad.

La metodología de proceso propuesta se basa en el sistema de la Figura 2. Se transmite un conjunto de 12 imágenes médicas provenientes de la digitalización de placas radiológicas, en formato DICOM, de tamaños 2048 x 2048, y profundidad de 8 bits. Cada imagen se comprime utilizando la técnica JPEG, con índice de calidad $Q_{i}(\mathrm{O})$. El valor $L_{i}(0)$ se calcula a partir del estimador de segundo orden resultante del codificador JPEG, de acuerdo al valor de calidad determinado por una evaluación preliminar. Para este trabajo, se considera el valor de calidad de $Q_{i}(0)=85$. Luego se promedian los valores de las medidas utilizadas. Para EBCOT se determinan $n_{q}$ niveles de calidad, de tal manera que existan $n_{q}$ valores de la tasa de compresión $L\left(n_{q}\right)$ para cada nivel de calidad $Q\left(n_{q}\right)$ y de modo que:

$$
\begin{aligned}
& \min \left\{L\left(n_{q}\right)\right\}<L_{o}<\max \left\{L\left(n_{q}\right)\right\} \\
& \min \left\{L\left(n_{q}\right)\right\}-\max \left\{L\left(n_{q}\right)\right\}=\mu
\end{aligned}
$$


Tabla 1. Valores promedio de las tasas de compresión calculadas para valores $Q_{i}(0)$ en JPEG, utilizando el modelo de segungo orden (SMO).

\begin{tabular}{|c|cccccccccc|}
\hline$Q_{i}(0)$ & 95 & $\mathbf{8 5}$ & 75 & 65 & 55 & 45 & 35 & 25 & 15 & 5 \\
\hline$L_{i}(0)(\mathrm{bpp})$ & 0.475 & $\mathbf{0 . 4 2 5}$ & 0.375 & 0.325 & 0.275 & 0.225 & 0.175 & 0.125 & 0.075 & 0.025 \\
\hline
\end{tabular}

Tabla 2. Valores promedio de la medida $M_{1}$ (error cuadrático medio) para las tres técnicas consideradas.

\begin{tabular}{|l|llllllllll|}
\hline$L_{i}(0)$ & 0.025 & 0.075 & 0.125 & 0.175 & 0.225 & 0.275 & 0.325 & 0.375 & $\mathbf{0 . 4 2 5}$ & 0.475 \\
\hline JPEG & 0.266 & 0.066 & 0.039 & 0.028 & 0.022 & 0.019 & 0.015 & 0.012 & $\mathbf{0 . 0 0 8}$ & 0.004 \\
EBCOT & 0.207 & 0.064 & 0.038 & 0.026 & 0.021 & 0.017 & 0.014 & 0.012 & $\mathbf{0 . 0 1 1}$ & 0.010 \\
SPIHT & 0.154 & 0.058 & 0.035 & 0.025 & 0.020 & 0.016 & 0.014 & 0.012 & $\mathbf{0 . 0 1 0}$ & 0.009 \\
\hline
\end{tabular}

donde $\mu$ es un valor definido de tal forma que los valores de las medidas sean cercanas. Para este trabajo, $n_{q}=5$. Una métrica de distorsión basada en la compresión de la fuente $D(R)$ se presenta incluida en los codificadores para EBCOT como parte de su sistema de estimación de la tasa. Se utiliza generalmente la relación señal a ruido (SNR), tal como lo proponen Taubman (2000) y Kosheleva (2004). Para la compresión se utilizaron las aplicaciones kadaku para JPEG2000, jpeglib para JPEG y la desarrollada por Said \& Pearlman (1996) para SPIHT. Para establecer la validación de la ergodicidad del valor medio de las métricas, se hará la evaluación para 6 y 24 imágenes médicas.

\section{Resultados y discusión}

En la Tabla 1 se ilustran los valores de la tasa de compresión promedio (estimados usando JPEG) para cada valor de calidad $Q_{i}(0)$. Los valores de las tasas promedio $L_{i}(0)$ estimadas se calcularon a partir del valor de calidad $Q_{i}(0)$ y el estimador de segundo orden.

En las Tablas 2-6 se ilustran las medidas promedio realizadas a las imágenes a ser transmitidas para las tres técnicas. Se observa que las curvas de distorsión generadas por la técnica JPEG se aproximan a las generadas por las técnicas SPIHT y EBCOT, lo que permite establecer el estimador de segundo orden como un buen estimador.

En la Figura 3 se observa una zona de alta correlación entre las métricas $M_{1}$ y $M_{2}$ que corresponde a la región de tasas de compresión $L\left(n_{q}\right)$ definida en la Tabla 7. En la Figura 4 se ilustra uno de los resultados del escenario de transmisión, en donde una imagen de 2048 x 2048 elementos es transmitida con 5 niveles de calidad. En la Tabla 8 se ilustran las tasas de compresión resultantes, lo que comprueba la reducción de los tiempos de transmisión.

En la Tabla 8 se observa que la técnica EBCOT puede obtener igual calidad perceptual de la imagen, utilizando una tasa de compresión menor a la calculada, y comprueba lo descrito por Taubman (2000). Sin embargo, es más claro observar la correlación existente para las medidas, utilizando la Figura 3.

Los valores estimados establecen una equivalencia entre los valores dados de calidad de imagen de una técnica y otra. Además, se pueden establecer curvas de aproximación entre métricas objetivas y subjetivas. Lo anterior permite utilizar técnicas de estimación de la calidad con base en conceptos de expertos.

El hecho de utilizar una métrica espectral basada en onduletas, implica que no se puede establecer una comparación con respecto a la técnica JPEG, debido a las diferentes bases utilizadas. Adicionalmente, para efectos de este trabajo, la métrica no es monotónica. En efecto, no se puede establecer un valor ergódico para cierto valor de la tasa de compresión. Lo anterior implica que se debe obtener una medida cuya base tenga una aproximación al filtro utilizado en la transformación. 
Ingeniería y Competitividad, Volumen 10, No.1, p. 73 - 83 (2008)

Tabla 3. Valores promedio de la relación señal a ruido (SNR) para las tres técnicas consideradas.

\begin{tabular}{|c|cccccccccc|}
\hline$L_{i}(0)$ & 0.025 & 0.075 & 0.125 & 0.175 & 0.225 & 0.275 & 0.325 & 0.375 & $\mathbf{0 . 4 2 5}$ & 0.475 \\
\hline JPEG & 59.714 & 71.809 & 76.502 & 79.332 & 81.244 & 82.855 & 84.677 & 86.848 & $\mathbf{9 0 . 1 1 9}$ & 96.811 \\
EBCOT & 62.739 & 72.660 & 76.984 & 80.024 & 82.135 & 83.854 & 85.278 & 86.449 & $\mathbf{8 7 . 5 0 7}$ & 88.524 \\
SPIHT & 65.386 & 73.559 & 77.687 & 80.477 & 82.548 & 84.275 & 85.667 & 86.864 & $\mathbf{8 7 . 9 3 1}$ & 88.911 \\
\hline
\end{tabular}

Tabla 4. Valores promedio de la medida $M_{2}$ (distancia de Czekanowski) para las tres técnicas consideradas.

\begin{tabular}{|c|cccccccccc|}
\hline$L_{i}(0)$ & 0.025 & 0.075 & 0.125 & 0.175 & 0.225 & 0.275 & 0.325 & 0.375 & $\mathbf{0 . 4 2 5}$ & 0.475 \\
\hline JPEG & 0.053 & 0.029 & 0.024 & 0.018 & 0.016 & 0.016 & 0.013 & 0.011 & $\mathbf{0 . 0 0 9}$ & 0.006 \\
EBCOT & 0.043 & 0.026 & 0.021 & 0.018 & 0.015 & 0.014 & 0.013 & 0.012 & $\mathbf{0 . 0 1 1}$ & 0.011 \\
SPIHT & 0.038 & 0.025 & 0.020 & 0.017 & 0.015 & 0.014 & 0.013 & 0.012 & $\mathbf{0 . 0 1 1}$ & 0.011 \\
\hline
\end{tabular}

Tabla 5. Valores promedio de la medida $M_{3}$ (fidelidad de la imagen) para las tres técnicas consideradas.

\begin{tabular}{|c|cccccccccc|}
\hline$L_{i}(0)$ & 0.025 & 0.075 & 0.125 & 0.175 & 0.225 & 0.275 & 0.325 & 0.375 & $\mathbf{0 . 4 2 5}$ & 0.475 \\
\hline JPEG & 0.9974 & 0.9994 & 0.9996 & 0.9997 & 0.9998 & 0.9998 & 0.9999 & 0.9999 & $\mathbf{0 . 9 9 9 9}$ & 0.99996 \\
EBCOT & 0.9981 & 0.9994 & 0.9996 & 0.9998 & 0.9998 & 0.9998 & 0.9999 & 0.9999 & $\mathbf{0 . 9 9 9 9}$ & 0.99991 \\
SPIHT & 0.9986 & 0.9995 & 0.9997 & 0.9998 & 0.9998 & 0.9998 & 0.9999 & 0.9999 & $\mathbf{0 . 9 9 9 9}$ & 0.99991 \\
\hline
\end{tabular}

Tabla 6. Valores promedio de la medida $M_{4}$ (medida espectral) para las tres técnicas consideradas.

\begin{tabular}{|c|cccccccccc|}
\hline$L_{\mathrm{i}}(0)$ & 0.025 & 0.075 & 0.125 & 0.175 & 0.225 & 0.275 & 0.325 & 0.375 & $\mathbf{0 . 4 2 5}$ & 0.475 \\
\hline JPEG & 197.158 & 31.168 & 12.182 & 14.382 & 10.182 & 4.992 & 7.198 & 1.792 & $\mathbf{1 . 4 0 0}$ & 1.000 \\
EBCOT & 44.872 & 77.968 & 4.592 & 4.598 & 1.198 & 2.398 & 0.798 & 1.400 & $\mathbf{1 . 4 0 0}$ & 2.000 \\
SPIHT & 32.328 & 4.200 & 5.600 & 0.998 & 1.598 & 2.198 & 2.598 & 4.798 & $\mathbf{6 . 3 8 2}$ & 6.382 \\
\hline
\end{tabular}

Tabla 7. Intervalos para la tasa de compresión determinada a partir de la región de alta correlación entre medidas.

\begin{tabular}{|cccc|}
\hline Medida & Valor mínimo & Valor máximo & Bits / pixel (bpp) \\
\hline $\mathrm{M}_{1}$ & 0.009 & 0.014 & $(0.475,0.275)$ \\
$\mathrm{M}_{2}$ & 0.010 & 0.018 & $(0.475,0.275)$ \\
\hline
\end{tabular}


Tabla 8. Tamaños de cada elemento de la imagen y tiempos de transmisión $R_{i}=4 \mathrm{~kb} / \mathrm{s}$.

\begin{tabular}{|c|cc|}
\hline$X_{i}$ & $L_{i}(\mathrm{bpp})$ & Tiempo de transmisión $(\mathrm{ms})$ \\
\hline Fuente & 8.0000 & 2.000 \\
$J P E G Q_{i}=85$ & $\mathbf{6 . 5 0 0 0}$ & $\mathbf{1 . 6 2 5}$ \\
$E B C O T n_{q}=1$ & $\mathbf{0 . 2 3 2 4}$ & $\mathbf{0 . 0 5 8}$ \\
EBCOT $n_{q}=2$ & 0.2776 & 0.069 \\
EBCOT $n_{q}=3$ & 0.3314 & 0.082 \\
EBCOT $n_{q}=4$ & 0.3908 & 0.097 \\
EBCOT $n_{q}=5$ & 0.4730 & 0.118 \\
\hline
\end{tabular}

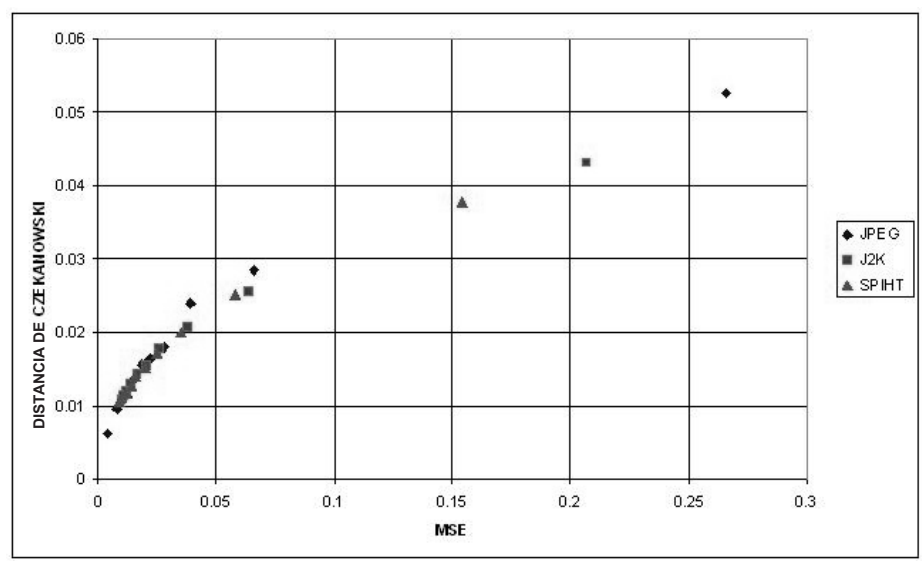

Figura 3. Correlación de métricas de calidad de imágenes para las técnicas JPEG, PSIHT y EBCOT.

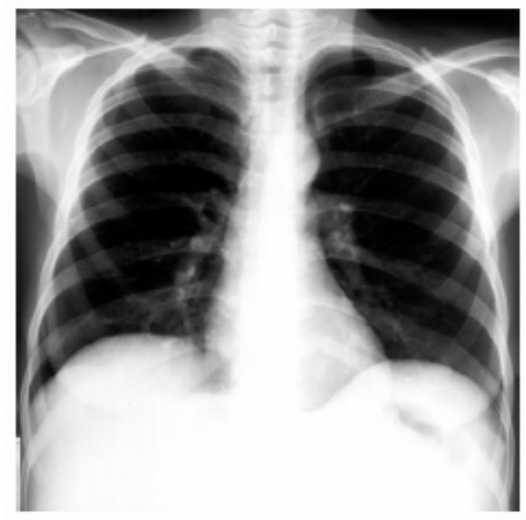

(a) Imagen Fuente $X_{i}$
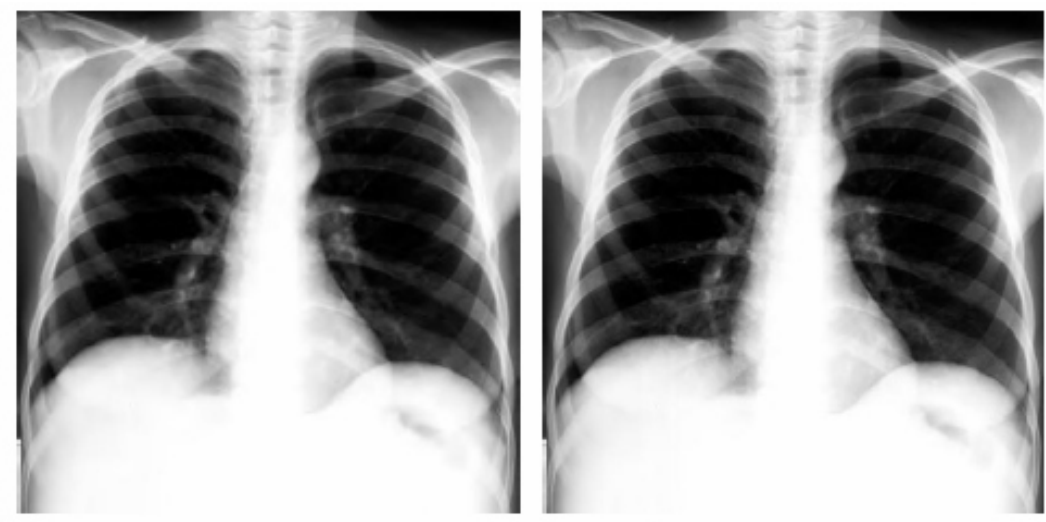

(b) Imagen transmitida usando EBCOT, (c) Imagen transmitida usando EBCOT, primera visualización $L(1)=0.2324$ bpp última visualización $L(5)=0.4730$ bpp

Figura 4. Visualización de una imagen transmitida con el método de estimación propuesto. 


\section{Conclusiones}

Se ha desarrollado un método de estimación de tasas de compresión basado en medidas y aplicado a un sistema de transmisión de imágenes. La ventaja obtenida con este método es la de reducir los tiempos de transmisión, utilizando la estimación de la tasa de una técnica de compresión rápida, pero con pérdidas de calidad, y aplicándola a una técnica de compresión avanzada, de tal forma que la calidad se mantenga para un tiempo más corto. Por lo tanto, para el caso de las imágenes médicas, no es necesario realizar nuevos estudios de evaluación de expertos en el momento de cambiar de tecnología, debido especialmente a muchos de los estudios de distorsión para JPEG hechos por Slone et al. (2003) y Zhu \& Wu (2005).

Para que el modelo de estimación de la tasa se ajuste a la transmisión de las imágenes, es necesaria la caracterización de éstas, debido a las propiedades de alta correlación que presentan. Lo anterior implica que es posible extraer una estructura de la imagen de modo que se pueda determinar si puede o no ser enviada en un tiempo más corto. Este método de estimación funciona para los procesos de compresión basados en métricas y orientados a la transmisión de imágenes de alta definición. Lo anterior incluye el uso de índices de calidad para la visualización remota de una cantidad masiva de imágenes o para una imagen de alta definición, con regiones de interés y sub-bandas.

Se ha definido un valor de tasa de compresión para cada técnica de compresión utilizada en la norma DICOM, bajo un valor de calidad de imagen definido. Al correlacionar las diferentes métricas, se han obtenido regiones donde se puede representar el valor de calidad JPEG con las diferentes métricas, sin requerir de una nueva evaluación de expertos.

Se requiere el análisis de la calidad de las imágenes y el control de la tasa de transmisión, mediante métricas basadas en el sistema visual humano.

\section{Referencias bibliográficas}

Aminlou, A., \& Fatemi, O. (2005). A novel efficient rate control algorithm for hardware implementation in JPEG2000. In Proceedings of the 2005 IEEE International Conference on Acoustics, Speech, and Signal Processing (ICASSP'05), Vol. 5, p.21-24.

Avcibas, I., Memon, N., \& Sankur, B. (2003). Steganalysis using image quality metrics. IEEE Transactions on Image Processing 12 (2), 221-229.

http://www.busim.ee.boun.edu.tr/ sankur/ SankurFolder/Image_Stego.pdf

Azpiroz-Leehan, J., \& Lerallut, J. F. (2006). JPEG2000 vs. full frame wavelet packet compression for smart card medical records. In Proceedings of the 28th IEEE EMBS Annual International Conference, New York, p. 2597-2600.

Chen, Y., \& Hao, P. (2004). Integer reversible transformation to make JPEG lossless. In Proceedings of the Seventh International Conference on Signal Processing (ICSP'04), p. 835-838.

Christopoulos, C. A., Ebrahimi, T., \& Skodas, A.N. (2000). JPEG2000: the new still picture compression standard. In Proceedings of the 2000 ACM Workshops on Multimedia, p. 45-49.

Dosselmann, R., \& Yang, X.D. (2005). Existing and emerging image quality metrics. In Proceedings of the 2005 Canadian Conference on Electrical and Computer Engineering, p. 1906-1913.

Dudek, G., Borys, P., \& Grzywna, Z. J. (2007). Lossy dictionary-based image compression method. Image Vision Computing 25 (6), 883-889.

Gangadhar, M., \& Bhatia, D. (2005). FPGA based EBCOT architecture for JPEG 2000. Microprocessors and Microsystems 29 (8-9), 363-373. 
Ginesu, G., Massidda, F. \& Giusto, D. (2006). A multi-factors approach for image quality assessment based on a human visual system model. Signal Processing: Image Communication $21(4), 316-333$.

Kosheleva, O. (2004). On the optimal choice of quality metric in image compression: a soft computing approach. Soft Computing 8(4), 268-273.

Kovacevic, J., \& Sweldens, W. (2000). Wavelet families of increasing order in arbitrary dimensions. IEEE Transactions on Image Processing 9(3), 480-496.

Krupinski, E.A., Johnson, J., Roehrig, H., Engstrom, M., Fan, J., Nafziger, J., Lubin, J., \& Dallas, W.J. (2003). Using a human visual system model to optimize soft-copy mammography display: influence of MTF compensation. Academic Radiology 10 (9), 1030-1035.

Lewis, A.S., \& Knowles, G. (1992). Image compression using the 2-D wavelet transform. IEEE Transactions on Image Processing 1 (2), 244-250.

López-Fernández, A. (2002). Reconstrucción bayesiana de imágenes SPECT usando modelos de Gauss-Markov. Estimación de parámetros. Tesis Doctoral, Universidad de Granada, Departamento de Lenguajes y Sistemas Informáticos, Granada, España.
Ringl, H., Schernthaner, R.E., Bankier, A.A., Weber, M., Prokop, M., Herold, C.J., \& SchaeferProkop, C. (2006). JPEG2000 compression of thin-section CT images of the lung: effect of compression ratio on image quality. Radiology 240 (3), 869-877.

Said A., \& Pearlman, W. A. (1996). An image multiresolution representation for lossless and lossy image compression. IEEE Transactions on Image Processing 5 (9), 1303-1310.

Salomon, D. (2004). Data compression: the complete reference. Third Edition, SpringerVerlag.

Shannon, C. E., \& Weaver, W. (1949). The mathematical theory of communication. University of Illinois Press.

Slone, R. M., Muka, E., \& Pilgram T.K. (2003). Irreversible JPEG compression of digital chest radiographs for primary interpretation: assessment of visually lossless threshold. Radiology 228 (2), 425-429.

Taubman, D. (2000). High performance scalable image compression with EBCOT. IEEE Transactions on Image Processing 9 (7), 1158-1170.

Zhu, H., \& Wu, H. (2005). New paradigm for compressed image quality metric: exploring band similarity with CSF and mutual information. In Proceedings of the 2005 IEEE International Geoscience and Remote Sensing Symposium (IGARSS'05), p. 696-699. 